\title{
Optimal Bidding Strategies of Wind-Thermal Power Producers
}

\author{
R. Laia ${ }^{1,2}$, H.M.I. Pousinho ${ }^{1}$, R. Melício ${ }^{1,2(\varpi)}$, and V.M.F. Mendes ${ }^{2,3}$ \\ ${ }^{1}$ IDMEC/LAETA, Instituto Superior Técnico, Universidade de Lisboa, Lisbon, Portugal \\ ruimelicio@gmail.com \\ ${ }^{2}$ Department de Física, Escola de Ciências e Tecnologia, Universidade de Évora, Évora, Portugal \\ ${ }^{3}$ Instituto Superior de Engenharia de Lisboa, Lisbon, Portugal
}

\begin{abstract}
This paper addresses a stochastic mixed-integer linear programming model for solving the self-scheduling problem of a thermal and wind power producer acting in an electricity market. Uncertainty on market prices and on wind power is modelled via a scenarios set. The mathematical formulation of thermal units takes into account variable and start-up costs and operational constraints like: ramp up/down limits and minimum up/down time limits. A mixed-integer linear formulation is used to obtain the offering strategies of the coordinated production of thermal and wind energy generation, aiming the profit maximization. Finally, a case study is presented and results are discussed.
\end{abstract}

Keywords: Mixed-integer linear programming · Stochastic optimization · Windthermal coordination . Offering strategies

\section{Introduction}

The adverse environmental impact of fossil fuel burning and the desire to reach energy supply sustainability promote exploitation of renewable sources. Mechanisms and policies provide subsidy and incentive for renewable energy conversion into electric energy [1], for instance, wind power conversion. But as the wind power technology matures and achieves breakeven costs, subsidy is due to be less significant and wind power conversion has to face the electricity markets for better profit [2]. Also, the incentives for wind power exploitation are feasible for low penetration levels but will become flawed as wind power integration rises [3]. EU in 2014 has of all new renewable installations a $43.7 \%$ based on wind power and is the seventh year running that over $55 \%$ of all additional power capacity is form renewable energy [4]. The growing worldwide usage of renewable energy is a fact, but electricity supply is still significantly dependent on fossil fuel burning, for instance, statistics for electricity supply in 2012 accounts that the usage of fossil fuel burning is more than $60 \%$ [5].

Deregulation of electricity market imposes that a generation company (GENCO) has to face competition to obtain the economic revenue. Periodic nodal variations of electricity prices [6] have to be taken into consideration. The wind power producer (WiPP) has to address wind power and electricity price uncertainties to decide for realistic bids, 
because cost is owed either in case of high bids due to the fact that other power producers must decrease or augment production to offset the deviation [7]. Thermal power producer has to address only electricity price uncertainty.

This paper focus on the coordinated trading of wind and thermal energy in order to achieve the optimal bidding strategies that provides the maximum profit. In the case study are matched the results from uncoordinated model with the results from the coordinated model.

\section{Technological Innovation for Cyber-Physical Systems}

Cyber-physical systems (CyPS) are systems whose operation is managed by a computing and communication core [8]. CyPS can be defined as smart systems that include computational and physical modules, effortlessly combined and strictly cooperating to sense the changing state of the real world [9].

On a first stage, cloud-based solutions can support the processing of models for helping trading in a pool-based electricity market so as to take more benefits of bids. Among these models the ones for the solution of the problems concerning with energy management and energy offers are specific vital for safeguarding a Wind-Thermal Power Producer (WTPP) business. The models for solving these problems are restricted by the computational resources, i.e., details about some reality are not considered in view of the extreme usage of computational requirements.

On a second stage, CyPS will make possible to connect the physical world, actuators and sensors, allowing the execution of the outputs of the systems decisions operating at a higher level. The strategy defined concerning with the commitment of thermal units (ThU) or the offers to the energy market can be implemented in real time with the CyPS.

\section{State of the Art}

Thermal energy conversion into electric energy has a significant state of art on optimization methods for solving the thermal scheduling problem $(\mathrm{ScP})$, ranging from the old priorities list method to the traditional mathematical methods up to the more lately reported artificial intelligence methods [10]. The priority list method is easily implemented and requires a small processing time, but does not guarantee an appropriate solution near the global optimal one [11]. In the classical methods are considered dynamic programming (DyP) and Lagrangian relaxation-based (LR) methods [12]. DyP method is a flexible one but has a limitation known by the "curse of dimensionality". The LR can overwhelmed the aforementioned limitation, but does not necessarily lead to a feasible solution, implying further processing for satisfying the violated constraints so as find a feasible solution, which does not ensure optimal solution. The mixed integer linear programming (MILP) method is used with success for solving the thermal ScP [13]. MILP is a widely used method for ScPs due to the tractability and extensive modeling capability [14]. Although, artificial intelligence methods based on neural networks, evolutionary algorithms and simulating annealing have been used, the main drawback of the artificial intelligence methods concerning with the possibility to obtain 
a solution near the global optimum one is a disadvantage. So, classical methods are the main methods in use as long as the functions describing the mathematical model have conveniently smoothness.

Deregulated market and variability of the source of wind power impose uncertainties to WiPP. These uncertainties have to be conveniently considered, i.e., processed into the variables of the problems [15] to be treated by a WiPP in order to identify how much to produce and the price for bidding.

A WiPP in a deregulated market can benefit without depending on third-parties from: a coordination of wind power production with energy storage technology [16]; a financial options as a tool for WiPP to hedge against wind power Unc [17]; a stochastic model envisioned to determine optimal offer strategies for WiPP participating in a day-ahead $(\mathrm{DaH})$ electricity market [18]. The stochastic model is a formulation explicitly taking into account the uncertainties tackled by the ScP of a WiPP [19], using multiple scenarios obtained by computer applications for wind power and market price forecasts [20].

\section{Problem Formulation}

\subsection{Wind Power Producer}

The uncertainties about the availability of wind power may imply differences between the energy traded with a WiPP and the actual quantity of energy supplied by the WiPP. The revenue $H_{x}$ of the GENCO for period $x$ is stated as:

$$
H_{x}=\vartheta_{x}^{D} I_{x}^{\text {offer }}+R_{x}
$$

In (1), $\vartheta_{x}^{D}$ is the energy price at period $x, I_{x}^{\text {offer }}$ is the power at the close of the DaH electricity market accepted to be traded and $R_{x}$ is the imbalance income derived from the balancing penalty of not acting in accordance with the accepted trade. The total deviation for period $t$ is stated as:

$$
\Theta_{x}=I_{x}^{a c t}-I_{x}^{\text {offer }}
$$

Where $I_{x}^{a c t}$ is the actual power for period $x$.

In (2), a positive deviation corresponds to the actual power traded higher than the traded in the DaH electricity market and a negative deviation corresponds to the power lower than the traded. Let $\vartheta_{x}^{+}$be the price paid for surplus of production and $\vartheta_{t}^{-}$the price to be charged for scarcity of production. Consider the price ratios given by the equalities stated as:

$$
h_{x}^{+}=\frac{\vartheta_{x}^{+}}{\vartheta_{x}^{D}}, h_{x}^{+} \leq 1 \text { and } h_{x}^{-}=\frac{\vartheta_{x}^{-}}{\vartheta_{x}^{D}}, h_{x}^{-} \geq 1
$$

In (3), the inequalities at the right of the equalities mean, respectively, that the positive deviation never has a higher price of penalization and the negative one never has a lower price of penalization in comparison with the value of the closing price. 


\subsection{Thermal Power Producer}

The operating cost, $A_{\phi r x}$, for a ThU is stated as:

$$
A_{\phi r x}=F_{r} v_{\phi r x}+b_{\phi r x}+d_{\phi r x}+Z_{r} c_{\phi r x} \quad \forall \phi, \quad \forall r, \quad \forall x
$$

In (4), $A_{\phi r x}$ is the operational cost for scenario $\phi$ of the ThU i at period $x . A_{\phi r x}$ is the sum of: the fixed production cost, $F_{r}$, a fixed associated with the unit state of operation; the added variable cost, $b_{\phi r x}$, part of this cost is associated with the quantity of fossil fuel used by the unit; and the start-up (SU) and shut-down (SD) costs, respectively, $d_{\phi r x}$ and $Z_{r}$, of the unit. The last three costs are in general described by nonlinear function and worse than that some of the functions are non- convex and non-differentiable functions, but some kind of smoothness is expected and required to use MILP, for instance, as being subdifferentiable functions.

The functions used to quantify the variable, the SU and SD costs of ThU in (4) are considered to be such that is possible to approximate those function by a piecewise linear or step functions. The variable $\operatorname{cost}, b_{\phi r x}$, is stated as:

$$
\begin{aligned}
& b_{\phi r x}=\sum_{q=1}^{Q} A_{r}^{q} \varsigma_{\phi r \mathrm{x}}^{q} \quad \forall \phi, \quad \forall r, \quad \forall x \\
& i_{\phi r \mathrm{x}}=i_{r}^{\min } v_{\phi \mathrm{r} x}+\sum_{q=1}^{Q} \varsigma_{\phi r x}^{q} \quad \forall \phi, \quad \forall r, \quad \forall x \\
& \left(X_{r}^{1}-i_{r}^{\min }\right) x_{\phi r x}^{1} \leq \varsigma_{\phi r x}^{1} \quad \forall \phi, \quad \forall r, \quad \forall x \\
& \varsigma_{\phi r x}^{1} \leq\left(X_{r}^{1}-i_{r}^{\min }\right) v_{\phi r x} \quad \forall \phi, \quad \forall r, \quad \forall x \\
& \left(X_{r}^{q}-X_{r}^{q-1}\right) x_{\phi r x}^{q} \leq \varsigma_{\phi r x}^{q} \quad \forall \phi, \quad \forall r, \quad \forall x, \quad \forall q=2, \ldots, Q-1 \\
& \varsigma_{\phi r x}^{q} \leq\left(X_{r}^{q}-X_{r}^{q-1}\right) x_{\phi r x}^{q-1} \quad \forall \phi, \quad \forall r, \quad \forall x, \quad \forall q=2, \ldots, Q-1 \\
& 0 \leq \varsigma_{\phi r x}^{Q} \leq\left(i_{r}^{\max }-X_{\phi r x}^{Q-1}\right) x_{\phi r x}^{Q-1} \quad \forall \phi, \quad \forall r, \quad \forall x
\end{aligned}
$$

In (5), the variable cost function is given by the sum of the product of the slope of each block, $A_{r}^{q}$, by the block power $\varsigma_{\phi r x}^{q}$. In (6), the power of the ThU is given by the minimum power production plus the summation of the block powers related with each block. The $0 / 1$ variable $v_{\phi r x}$ guarantees that the power production is 0 if the ThU is in the state offline. In (7), if the $0 / 1$ variable $x_{\phi r x}^{q}$ is equal to 0 , then the block power $\varsigma_{\phi r x}^{1}$ can be lower than the block 1 maximum power; otherwise and in conjunction with (8), if the ThU is in the state on, then $\varsigma_{\phi r x}^{1}$ is equal to the block 1 maximum power. In (9), from the second bock to the second last one, if the $0 / 1 x_{\phi r x}^{q}$ is 0 , then the block power 
$\varsigma_{\phi r x}^{q}$ can be lower than the block $q$ maximum power; otherwise and in conjunction with (10), if the ThU is in the state on, then $\varsigma_{\phi r x}^{q}$ is equal to the block $q$ maximum power. In (11), the block power must be between 0 and the last block maximum power.

The nonlinearities of the start-up costs, $d_{\phi r x}$, is normally considered to be described by an exponential function. This exponential function is estimated by a piecewise linear formulation as in [2] stated as:

$$
d_{\phi r x} \geq K_{r}^{\sigma}\left(v_{\phi r x}-\sum_{h=1}^{\sigma} v_{\phi r x-h}\right) \quad \forall \phi, \quad \forall r, \quad \forall x
$$

In (12), the second term models the lost of ThU, i.e., if the unit is a case of being in the state online at period $x$ and has been in the state offline in the $\sigma$ preceding periods, the term in parentheses is 1 . So, in such a case a SU cost is incurred for the thermal energy that are not accountable for added value in a sense of that energy has not been converted into electric energy. The maximum number for $\sigma$ is given by the number of periods need to cool down, i.e., completely lose all thermal energy. So, for every period at cooling and until total cooling one inequality like (12) is considered.

The units have to perform in accordance with technical constraints that limit the power between successive hours stated as:

$$
\begin{gathered}
i_{r}^{\min } v_{\phi r x} \leq i_{\phi r x} \leq i_{\phi r x}^{\max } \quad \forall \phi, \quad \forall r, \quad \forall x \\
i_{\phi r x}^{\max } \leq i_{r}^{\max }\left(v_{\phi r x}-c_{\phi r x+1}\right)+S D c_{\phi r x+1} \quad \forall \phi, \quad \forall r, \quad \forall x \\
i_{\phi r x}^{\max } \leq i_{\phi r x-1}^{\max }+R U v_{\phi r x-1}+S U y_{\phi r x} \quad \forall \phi, \quad \forall r, \quad \forall x \\
i_{\phi r x-1}-i_{\phi r x} \leq R D v_{\phi r x}+S D c_{\phi r x} \quad \forall \phi, \quad \forall r, \quad \forall x
\end{gathered}
$$

In (13) and (14), the upper bound of $i_{\phi r x}^{\max }$ is defined as being the maximum available power of the ThU. This variable is used to consider: actual capacity of the ThU, SU/SD ramp rate and ramp-up limits. In (16), the ramps-down and SD ramp rate limits are defined. In (14), (15) and (16), the relation between the SU and SD variables of the ThU are provided, using $0 / 1$ variables for describing the states and data parameters for rampdown, SD and ramp-up rate limits.

The minimum down time (DT) constraint is stated as:

$$
\sum_{x=1}^{J_{r}} v_{\phi r x}=0 \quad \forall \phi, \quad \forall r
$$

$$
\sum_{x=k}^{k+D X_{r}-1}\left(1-v_{\phi r x}\right) \geq D X_{r} c_{\phi r x} \quad \forall \phi, \quad \forall r, \quad \forall k=J_{r}+1 \ldots X-D X_{r}+1
$$




$$
\begin{gathered}
\sum_{x=k}^{X}\left(1-v_{\phi r x}-c_{\phi r x}\right) \geq 0 \quad \forall \phi, \quad \forall r, \quad \forall k=X-D X_{r}+2 \ldots X \\
J_{r}=\min \left\{X,\left(D X_{r}-s_{\phi r 0}\right)\left(1-v_{\phi r 0}\right)\right\}
\end{gathered}
$$

In (17), if the minimum DX is reached, then the unit will be offline at initial period. In (18), the minimum DX will be satisfied for all the sets of sequential periods of size $D X_{r}$. In (19), the minimum DX will be satisfied for the last $D X_{r}-1$ periods.

The minimum up time (UX) constraint is also forced by constraints stated as:

$$
\sum_{x=1}^{N_{r}}\left(1-v_{\phi r x}\right)=0 \quad \forall \phi, \quad \forall r
$$

$$
\begin{gathered}
\sum_{x=k}^{k+U X_{r}-1} v_{\phi r x} \geq U X_{r} y_{\phi r x} \quad \forall \phi, \quad \forall r, \quad \forall k=N_{r}+1 \ldots X-U X_{r}+1 \\
\sum_{x=k}^{X}\left(v_{\phi r x}-c_{\phi r x}\right) \geq 0 \quad \forall \phi, \quad \forall r, \quad \forall k=X-U X_{r}+2 \ldots X \\
N_{r}=\min \left\{X,\left(U X_{r}-V_{\phi r 0}\right) v_{\phi r 0}\right\}
\end{gathered}
$$

In (20), if the minimum UX is not reached, then the unit will be offline at initial period. In (21), the minimum UX will be satisfied for all the sets of sequential periods of size $U X_{r}$. In (22), the minimum UX will be satisfied for the last $U X_{r}-1$ periods.

The operational status of the ThU is stated as:

$$
\begin{gathered}
y_{\phi r x}-c_{\phi r x}=v_{\phi r x}-v_{\phi r x-1} \quad \forall \phi, \quad \forall r, \quad \forall x \\
y_{\phi r x}+c_{\phi r x} \leq 1 \quad \forall \phi, \quad \forall r, \quad \forall x
\end{gathered}
$$

The total power produced by the ThU is stated as:

$$
i_{\phi x}^{g}=\sum_{r=1}^{R} i_{\phi r x} \quad \forall \phi, \quad \forall x
$$

In (25), note by (13) to (16) and (23), (24) that if the unit is not in the state of online then the power of the unit is null.

\subsection{Objective Function}

The offer submitted by the GENCO, WTPP, is the summation of the power offered from the ThU and the power offered from the wind farm (WiF). The offer is stated as:

$$
i_{\phi x}^{o f f e r}=i_{\phi x}^{t h}+i_{\phi x}^{D} \quad \forall \phi, \quad \forall x
$$

The actual power produced by the GENCO is the summation of the power produced by ThU and the power produced by the WF. The actual power is stated as: 


$$
i_{\phi x}^{a c t}=i_{\phi x}^{g}+i_{\phi x}^{\phi d} \quad \forall \phi, \quad \forall x
$$

In (27), $i_{\phi x}^{g}$ is the actual power produced by ThU and $i_{\phi x}^{\phi d}$ is the actual power produced by the WF for scenario $\phi$.

Consequently, the expected revenue of the GENCO is stated as:

$$
\sum_{\varphi=1}^{N_{\Phi}} \sum_{x=1}^{N_{X}} \eta_{\phi}\left[\left(\vartheta_{\phi x}^{D} P_{\phi x}^{\text {offer }}+\vartheta_{\phi x}^{D} h_{\phi x}^{+} \Theta_{\phi x}^{+}-\vartheta_{\phi t}^{D} h_{\phi x}^{-} \Theta_{\phi x}^{-}\right)-\sum_{r=1}^{R} A_{\phi r x}\right] \quad \forall \phi, \quad \forall x
$$

Subject to:

$$
\begin{gathered}
0 \leq i_{\phi x}^{\text {offer }} \leq i_{\phi x}^{M} \quad \forall \phi, \quad \forall x \\
\Theta_{x \phi}=\left(i_{\phi x}^{a c t}-i_{\phi x}^{o f f e r}\right) \quad \forall \phi, \quad \forall x \\
\Theta_{x \phi}=\Theta_{x \phi}^{+}-\Theta_{x \phi}^{-} \quad \forall \phi, \quad \forall x \\
0 \leq \Theta_{x \phi}^{+} \leq I_{x \phi} d_{x} \quad \forall \phi, \quad \forall x
\end{gathered}
$$

In (29), $i_{\phi x}^{M}$ is maximum available power, limited by the sum of the installed capacity in the $\mathrm{WF}, i^{E \mathrm{max}}$, with the maximum thermal production stated as:

$$
i_{\phi x}^{M}=\sum_{r=1}^{R} i_{\phi r x}^{\max }+i^{E \max } \quad \forall \phi, \quad \forall x
$$

Some system operators require non-decreasing offers to be submitted by the GENCO. Non-decreasing offers is considered by a constraint stated as:

$$
\left(i_{\phi x}^{\text {offer }}-i_{\phi^{\prime} x}^{\text {offer }}\right)\left(\vartheta_{\phi x}^{D}-\vartheta_{\phi^{\prime} x}^{D}\right) \geq 0 \quad \forall \phi, \phi^{\prime}, \quad \forall x
$$

In (29), if the increment in price in two successive hours is not null, then the increment in offers in the two successive hours has two be of the same sign of the increment in price or a null value.

\section{Case Study}

The proposed SMILP model is applied to a case study of a GENCO with a WTPP, having 8 units with a total installed capacity of $1440 \mathrm{MW}$, the data is in [22]. Data from the Iberian electricity market for 10 days of June 2014 [21] are used for the energy prices and the energy produced from WF. This data is depicted in Fig. 1. 

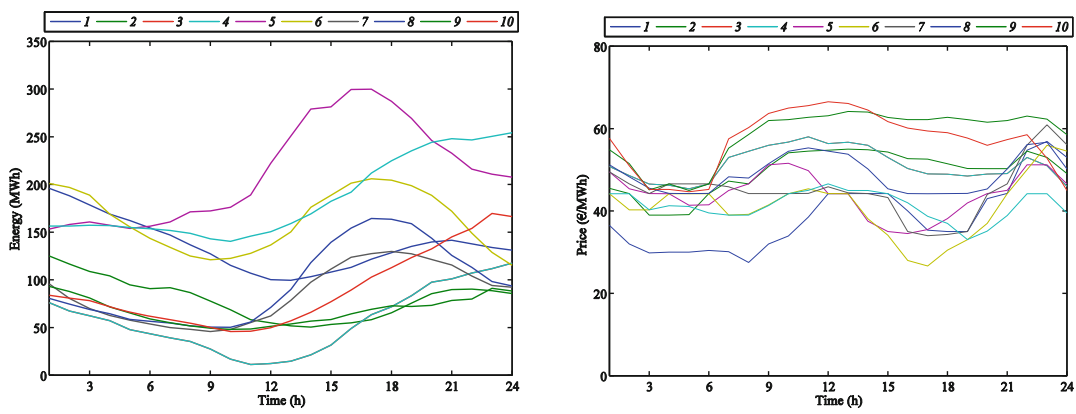

Fig. 1. Iberian electricity market June 2014 (ten days); left: prices, right: energy.

The non-decreasing offer is required. The energy produced is achieved through the total energy produced from wind scaled to the installed capacity in the WF, $360 \mathrm{MW}$. The expected results with and without coordination are depicted in Table 3.

Table 3. Results with and without coordination

\begin{tabular}{l|l|l}
\hline Case & Expected profit $(€)$ & Execution Time (s) \\
\hline Wind uncoordinated & 119200 & 0.02 \\
\hline Thermal uncoordinated & 516848 & 0.13 \\
\hline Coordinated wind and thermal & 642326 & 0.13 \\
\hline Gain $(\%)$ & 0,99 & - \\
\hline
\end{tabular}

The non-decreasing energy offer for hours 5 and 20 is depicted in Fig. 2.
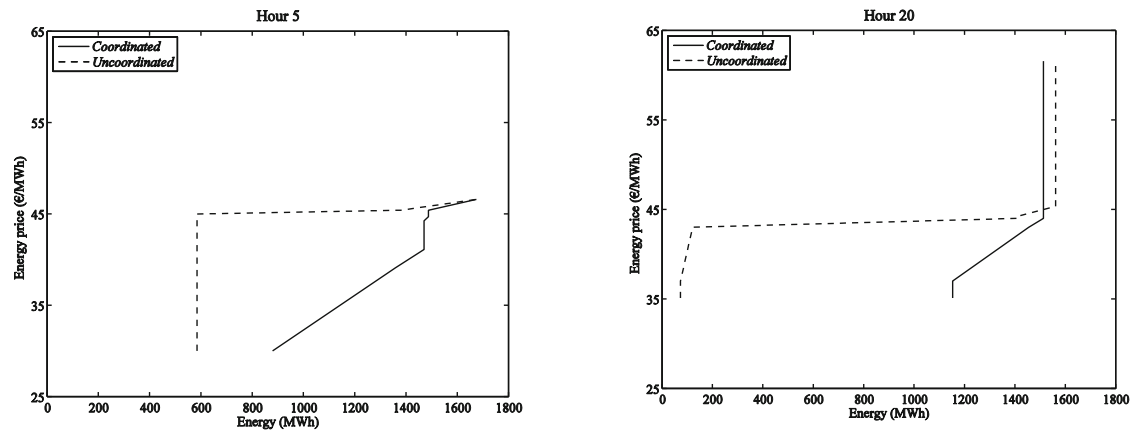

Fig. 2. Bidding energy offers.

In Fig. 2, the coordination allows for a minimum value of power offered higher than the one offered without coordination and allows for a lower price of the offering, which is a potential benefit to into operation. 


\section{Conclusion}

Cyber-physical systems can be a great advantage for helping power systems to accommodate the changing state of the real world. Particularly, a contribution is given in this paper in what concerns the biding in the day-ahead electricity market for a thermal and wind power producer. A SMILP model for solving the offering strategy and the self$\mathrm{ScP}$ of a thermal and wind power producer is settled in this paper. A mixed-integer linear program is considered to formulate the operational features of ThU. The coordinated offer of thermal and wind power proved to provide better revenue results than the sum of the isolated offers. The stochastic programming is a appropriate model to address Unc in modeling through scenarios. So, the SMILP model demonstrated to be accurate as well computationally acceptable. Since the bids are made in the DaH electricity market, this proposed SMILP model is a useful tool for the power producer.

Acknowledgments. The work presented in this paper is funded through Portuguese Funds by the Foundation for Science and Technology-FCT for project scope LAETA 2015-2020, UID/EMS/50022/2013.

\section{References}

1. Kongnam, C., Nuchprayoon, S.: Feed-in tariff scheme for promoting wind energy generation. In: IEEE Bucharest Power Tech Conference, pp. 1-6 (2009)

2. Morales, J.M., Conejo, A.J., Ruiz, J.P.: Short-term trading for a wind power producer. IEEE Trans. Power Syst. 25(1), 554-564 (2010)

3. Bitar, E.Y., Rajagopal, R., Khargonekar, P.P., Poolla, K., Varaiya, P.: Bringing wind energy to market. IEEE Trans. Power Syst. 27(3), 1225-1235 (2012)

4. Pineda, I., Wilkes, J.: Wind in power: 2014 European statistics (2015)

5. Key World Energy Statistics Report. International Energy Agency (2014)

6. Wu, A., Shahidehpour, L., Li, T.: Stochastic security-constrained unit commitment. IEEE Trans. Power Syst. 22(2), 800-811 (2007)

7. Pousinho, H.M.I., Catalão, J.P.S., Mendes, V.M.F.: Offering strategies for a wind power producer considering uncertainty through a stochastic model. In: 12th International Conference on Probabilistic Methods Applied to Power Systems, pp. 1139-1144 (2012)

8. Rajkumar, R.: A cyber-physical future. Proc. IEEE 100, 1309-1312 (2012)

9. Foundations for innovation in cyber-physical systems. Workshop summary report. National Institute of Standards and Technology (2013)

10. Trivedi, A., Srinivasan, D., Biswas, S., Reindl, T.: Hybridizing genetic algorithm with differential evolution for solving the unit commitment scheduling problem. Swarm Evol. Comput. 23, 50-64 (2015)

11. Senjyu, T., Shimabukuro, K., Uezato, K., Funabashi, T.: A fast technique for unit commitment problem by extended priority list. IEEE Trans. Sustain. Energy 18, 277-287 (2003)

12. Laia, R., Pousinho, H.M.I., Melício, R., Mendes, V.M.F., Reis, A.H.: Schedule of thermal units with emissions in a spot electricity market. In: Camarinha-Matos, L.M., Tomic, S., Graça, P. (eds.) DoCEIS 2013. IFIP AICT, vol. 394, pp. 361-370. Springer, Heidelberg (2013)

13. Ostrowski, J., Anjos, M.F., Vannelli, A.: Tight mixed integer linear programming formulations for the unit commitment problem. IEEE Trans. Power Syst. 27(1), 39-46 (2012) 
14. Floudas, C., Lin, X.: Mixed integer linear programming in process scheduling: modeling, algorithms, and applications. Ann. Oper. Res. 139, 131-162 (2005)

15. El-Fouly, T.H.M., Zeineldin, H.H., El-Saadany, E.F., Salama, M.M.A.: Impact of wind generation control strategies, penetration level and installation location on electricity market prices. IET Renew. Power Gener. 2, 162-169 (2008)

16. Angarita, J.L., Usaola, J., Crespo, J.M.: Combined hydro-wind generation bids in a poolbased electricity market. Electr. Power Syst. Res. 79, 1038-1046 (2008)

17. Hedman, K., Sheble, G.: Comparing hedging methods for wind power: using pumped storage hydro units vs options purchasing. In: International Conference on Probabilistic Methods Applied to Power Systems, pp. 1-6 (2006)

18. Matevosyan, J., Soder, L.: Minimization of imbalance cost trading wind power on the shortterm power market. IEEE Trans. Power Syst. 21(3), 1396-1404 (2006)

19. Ruiz, P.A., Philbrick, C.R., Sauer, P.W.: Wind power day-ahead uncertainty management through stochastic unit commitment policies. In: IEEE/PES Power Systems Conference and Exposition, pp. 1-9 (2009)

20. Coelho, L.S., Santos, A.A.P.: A RBF neural network model with GARCH errors: application to electricity price forecasting. Electr. Power Syst. Res. 81, 74-83 (2011)

21. Red Eléctrica de España. (October 2015) http://www.esios.ree.es/web-publica/

22. Laia, R., Pousinho, H.M.I., Melício, R., Mendes, V.M.F.: Self-scheduling and bidding strategies of thermal units with stochastic emission constraints. Energy Convers. Manage. 89, 975-984 (2015) 